Pyatigorsk medical-pharmaceutical Institute-branch state budget educational institution of higher professional

p-ISSN: 2308-4944 (print) e-ISSN: 2409-0085 (online)

Year: $2018 \quad$ Issue: $01 \quad$ Volume: 57

Published: $30.01 .2018 \quad \underline{\text { http://T-Science.org }}$ education "Volgograd State Medical University" Ministry of healthcare of the Russian Federation, Pyatigorsk

M.K. Tsidaeva

Pyatigorsk medical-pharmaceutical Institute-branch state budget educational institution of higher professional education "Volgograd State Medical University" Ministry of healthcare of the Russian Federation, Pyatigorsk

SECTION 28. Pharmaceutical Sciences.

\title{
A STUDY OF THE LEVEL OF ORGANIZATION OF INPATIENT AND OUTPATIENT MEDICAL CARE TO PATIENTS WITH DIABETES IN THE REPUBLIC OF NORTH OSSETIA-ALANIA
}

Abstract: One of the main criteria for assessing quality of care is the generally accepted measure of patient satisfaction with appropriate medical services.

The study of satisfaction of patients with diabetes level of health care as one aspect of quality of care, allows you to select the main directions and ways of its improvement.

Key words: Pharmacoeconomics, pharmacoepidemiology, drug supply of the population, drug supply of rural population, pharmaceutical care.

Language: Russian

Citation: Airo IN, Tsidaeva MK (2018) A STUDY OF THE LEVEL OF ORGANIZATION OF INPATIENT AND OUTPATIENT MEDICAL CARE TO PATIENTS WITH DIABETES IN THE REPUBLIC OF NORTH OSSETIA-ALANIA. ISJ Theoretical \& Applied Science, 01 (57): 152-155.

Soi: http://s-o-i.org/1.1/TAS-01-57-27 Doi: crossef https://dx.doi.org/10.15863/TAS.2018.01.57.27

\section{ИССЛЕДОВАНИЕ УРОВНЯ ОРГАНИЗАЦИИ СТАЦИОНАРНОЙ И АМБУЛАТОРНОЙ МЕДИЦИНСКОЙ ПОМОЩИ БОЛЬНЫМ САХАРНЫМ ДИАБЕТОМ В РЕСПУБЛИКЕ СЕВЕРНАЯ ОСЕТИЯ-АЛАНИЯ}

\begin{abstract}
Аннотация: Одним из основных критериев оценки качества медицинской помощчи является общепризнанный показатель удовлетворенности пациентов деятельностью соответствующих медицинских служб.

Изучение степени удовлетворенности больных сахарным диабетом уровнем медицинского обслуживания, как одного из аспектов качества медицинской помощи, позволяет выделить главные направления и пути ее улучшения.

Ключевые слова: Фармакоэкономика, фармакоэпидемиология, лекарственное обеспечение населения, лекарственное обеспечение населения сельской местности, фармацевтическая помощь.
\end{abstract}

Цель нашего исследования: оценка качества организации стационарной и амбулаторной медицинской помощи больным сахарным диабетом в Республике Северная Осетия-Алания

Методы исследования: анкетирование, интервьюирование.

Результаты исследования:

Для выяснения мнения больных сахарным диабетом о положительных и отрицательных сторонах деятельности амбулаторнополиклинического и стационарного звена диабетологической службы, а также субъективной оценки больного своего состояния нами проведено социологическое исследование по разработанным нами анкетам.

Результаты нашего исследования свидетельствуют о снижении показателей положительной оценки и росте показателей неудовлетворительной оценки своего здоровья с возрастом респондентов. Прослежена связь частоты декомпенсации с контролем гликемии.

Контроль гликемии при ухудшении самочувствия проводят $49,5 \%$ респондентов, проводят ежедневно $35,3 \%$ и только $\quad 6,6 \%$ проводят контроль перед каждым приемом пищи. При этом 8,6\% респондентов совсем не проводят контроля гликемии. 


\begin{tabular}{|c|c|c|c|c|c|c|}
\hline Impact Factor: & $\begin{array}{l}\text { ISRA (India) } \\
\text { ISI (Dubai, UAE } \\
\text { GIF (Australia) } \\
\text { JIF }\end{array}$ & $\begin{array}{r}=1.344 \\
=0.829 \\
=0.564 \\
=1.500\end{array}$ & $\begin{array}{l}\text { SIS (USA) } \\
\text { PИHЦ (Russia) } \\
\text { ESJI (KZ) } \\
\text { SJIF (Morocco) }\end{array}$ & $\begin{array}{l}=0.912 \\
=0.207 \\
=4.102 \\
=2.031\end{array}$ & $\begin{array}{l}\text { ICV (Poland) } \\
\text { PIF (India) } \\
\text { IBI (India) }\end{array}$ & $\begin{array}{l}=6.630 \\
=1.940 \\
=4.260\end{array}$ \\
\hline
\end{tabular}

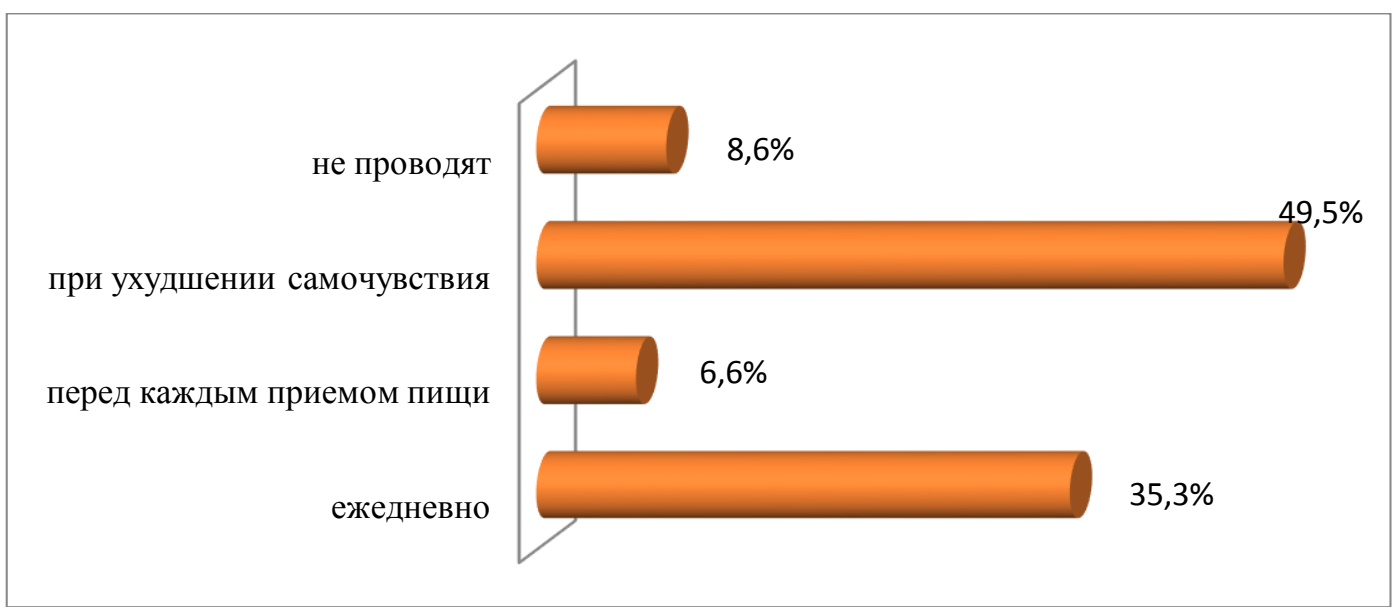

Рисунок 1 - Анализ контроля гликемии больных сахарным диабетом

Результаты исследования степени удовлетворенности амбулаторнополиклинической помощью показали, что около $40 \%$ респондентов не удовлетворены качеством медицинской помощи.

Основными причинами, по мнению респондентов, являются: уровень обследования (48\%), нерешенные организационные вопросы и чрезмерная загруженность врачей (24\%), отсутствие необходимых специалистов $(32,6 \%)$. При этом отмечается низкая эффективность профилактической работы в поликлинике, страдает информированность больных о заболевании и возможных его осложнениях. В результате отсутствует систематический самоконтроль уровня гликемии.

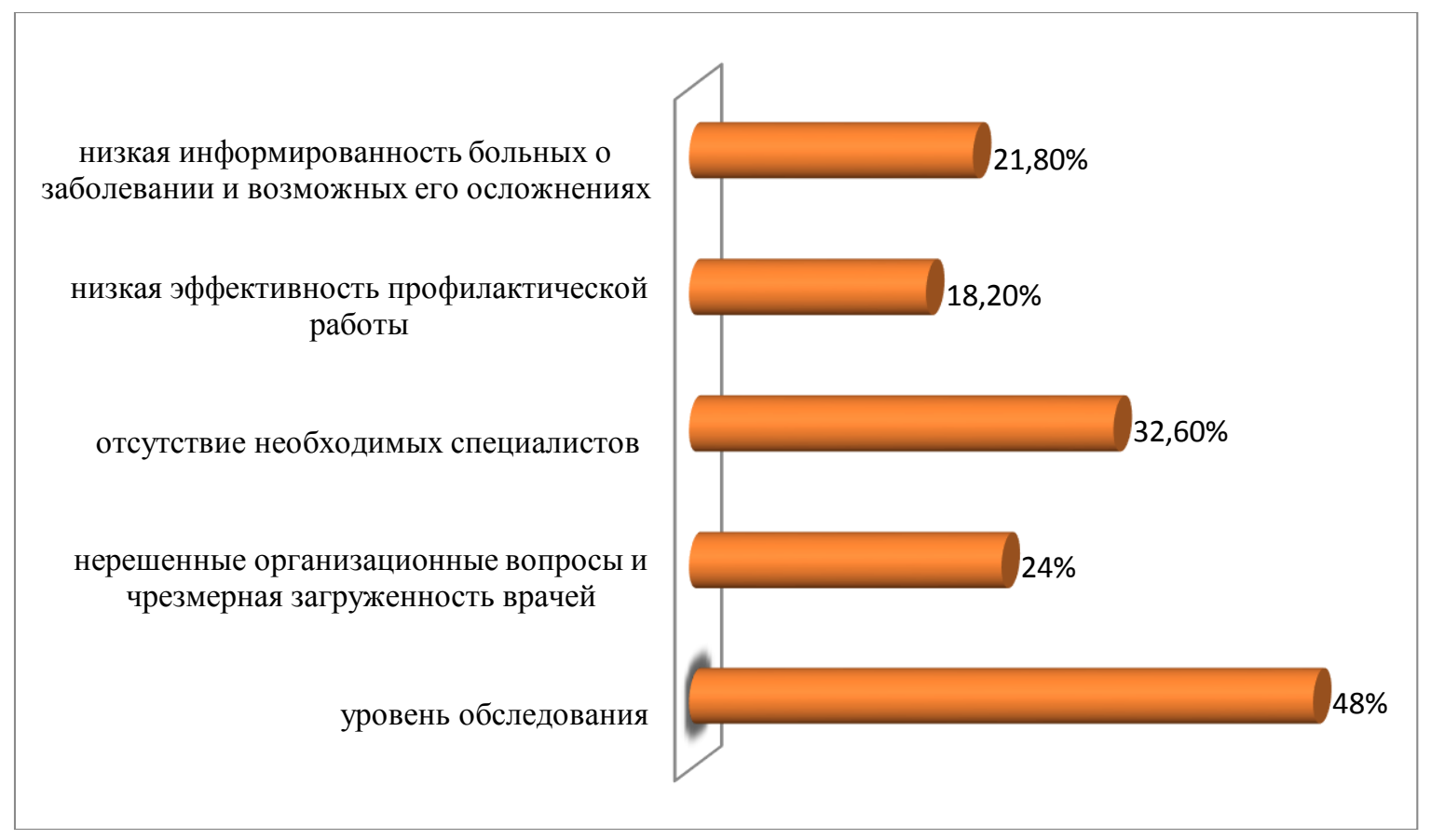

Рисунок 2 - Изучение степени удовлетворенности больных сахарным диабетом амбулаторнополиклинической помощью.

Анализ данных социологического исследования позволил также изучить степень удовлетворенности качеством стационарной медицинской помощи больных сахарным диабетом.
В целом, не удовлетворены стационарной медицинской помощью $50,6 \%$, респондентов. Очень высокий процент респондентов (79,7\%) указал на отсутствие в стационарах необходимых медикаментов, значительная часть которых приобретается самими пациентами. Во всех 
возрастных группах отмечается большой процент $(45,3 \%)$ неудовлетворенности пациентов качеством питания в стационаре.

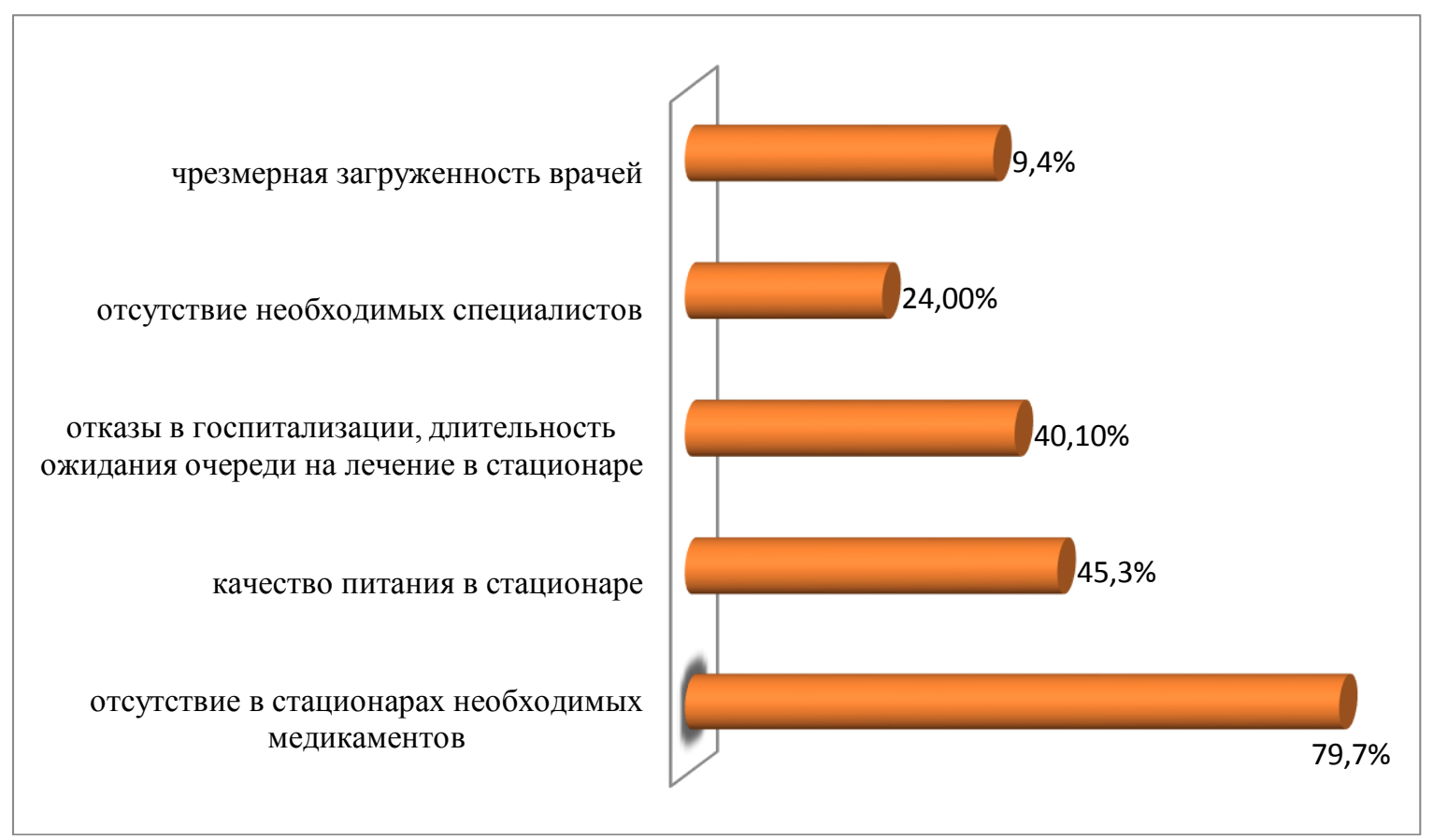

\section{Рисунок 3 - Изучение степени удовлетворенности больных сахарным диабетом стационарной} помощью.

Частота отказов в госпитализации, как и длительность ожидания очереди на лечение в стационаре, по данным анкетирования, указывают на отсутствие достаточного числа эндокринологических коек в республике, необходимых для удовлетворения потребности в госпитализации больных сахарным диабетом.

Неудовлетворенность в ряде случаев доступностью и качеством медицинского обслуживания диктует необходимость совершенствования работы медицинских организаций, а также безусловного соблюдения принципов медицинской этики и деонтологии.

Обобщая в целом материалы нашего исследования, следует отметить, что результаты, полученные на основании анализа мнения большинства респондентов, могут быть использованы при составлении перспективных программ развития и совершенствования медицинской помощи больным сахарным диабетом.

\section{Выводы:}

Таким образом, данные нашего исследования подтверждают высокую актуальность проблемы, связанную с заболеваемостью и медико-социальной значимостью сахарного диабета в Республике Северная Осетия-Алания. Снизить заболеваемость и частоту поздних осложнений возможно лишь при соответствующей организации всех звеньев диабетологической службы. Повышению качества и эффективности специализированной помощи больным сахарным диабетом будет способствовать модернизация управления системой медицинской помощи больным сахарным диабетом.

\section{References:}

1. (2018) Severo-Osetinskiy informatsionnyy portal «15-Y REGION». - [Elektronnyy resurs]. -Internet-portal Available: http://region15.ru (Accessed: 10.01.2018).
2. (2004) Vnutrennyaya kartina bolezni pri sakharnom diabete tipa 1 / P.I. Sidorov, I.A. Novikova, A.G. Solov'ev, N.N. Mul'kova // Problemy endokrinologii. 2004. - № 3. - p. 3-6. 


\begin{tabular}{l|lr|ll|ll} 
& ISRA (India) & $=\mathbf{1 . 3 4 4}$ & SIS (USA) & $=\mathbf{0 . 9 1 2}$ & ICV (Poland) & $=\mathbf{6 . 6 3 0}$ \\
Impact Factor: & ISI (Dubai, UAE) $=\mathbf{0 . 8 2 9}$ & PUH (Russia) $=\mathbf{0 . 2 0 7}$ & PIF (India) & $=\mathbf{1 . 9 4 0}$ \\
& GIF (Australia) & $=\mathbf{0 . 5 6 4}$ & ESJI (KZ) & $=4.102$ & IBI (India) & $=\mathbf{4 . 2 6 0}$ \\
& JIF & $=\mathbf{1 . 5 0 0}$ & SJIF (Morocco) & $=\mathbf{2 . 0 3 1}$ & & \\
\hline
\end{tabular}

3. Dedov I.I. (1998) Sakharnyy diabet u detey i podrostkov: rukovodstvo dlya vrachey / I. I. Dedov, T. JI. Kuraeva, M.: Izd-vo Bereg, 1998.

4. Dedov I.I. (1998) Vvedenie v diabetologiyu: ruk-vo dlya vrachey / I.I. Dedov, V.V. Fadeev. M.: Izd-vo Bereg, 1998. - 200 p.

5. (2006) DLO: otsenka ispol'zovaniya lekarstvennykh sredstv/ P.C. Safiullin, F.F. Yarkaeva, R.I. Yagudina i dr.// Novaya apteka. Effektiv. upravlenie. -2006-№ 10. -p.31 -33.

6. Dremova N.B. (1998) Kontseptsiya marketingovykh issledovaniy assortimenta lekarstvennykh sredstv $\mathrm{v}$ farmatsevticheskikh organizatsiyakh / N.B. Dremova, E.V. Lazareva // Ekon. vestn. farmatsii. 1998. - №12. - p. 6774.
7. Kasatkina E.P. (1996) Sakharnyy diabet u detey./ Kasatkina E.P. -M.1996. - p. 224-236.

8. Morozov V.A. (2017) Razrabotka metodicheskikh podkhodov $\mathrm{k}$ optimizatsii lekarstvennogo obespecheniya bol'nykh sakharnym diabetom Respubliki Severnaya Osetiya-Alaniya

9. (2002) Obespechenie gosudarstvennykh garantiy opredelennym gruppam naseleniya Respubliki Buryatiya B.G. Bal'zhirov [i dr.] Novaya Apteka. 2002. -p. 17-21.

10. (2012) Prikaz Minzdrava Rossii ot 09.11.2012 $\mathrm{N} 750 \mathrm{n}$ "Ob utverzhdenii standarta pervichnoy mediko-sanitarnoy pomoshchi detyam pri insulinzavisimom sakharnom diabete" 